\title{
Participatory design of agricultural decision support tools: taking account of the use situations
}

\author{
Marianne Cerf • Marie-Hélène Jeuffroy • Lorène Prost • \\ Jean-Marc Meynard
}

\begin{abstract}
Accepted: 8 March 2012 / Published online: 4 April 2012
(C) INRA and Springer-Verlag, France 2012
\end{abstract}

\begin{abstract}
Although many agronomic researchers currently focus on designing and developing decision support systems, they rarely discuss the methodological implications of such work. In this paper, with the examples of two decision support systems, we propose methodological elements for conducting the participatory design of such tools. Our proposition aims at building dialogue between designers and users but also between humans, tools and work situations. We focus on two main stages: first, a diagnosis of the uses of decision tools within current working situations and, second, the use of a prototype of the tool under design. The first stage serves to characterize the diversity of uses and user situations in order to determine the tool's flexibility and to identify new concepts for tools. The second stage involves setting up an arrangement whereby a prototype of the decision support tool, open to
\end{abstract}

\footnotetext{
M. Cerf $(\bowtie) \cdot$ L. Prost

UR1326 Sciences en Société, INRA,

77420 Champs-sur-Marne, France

e-mail: cerf@agroparistech.fr

L. Prost

e-mail: prost@grignon.inra.fr

M.-H. Jeuffroy

UMR211 Agronomie, INRA,

78850 Thiverval-Grignon, France

e-mail: jeuffroy@grignon.inra.fr

M.-H. Jeuffroy

UMR Agronomie, AgroParisTech,

78850 Thiverval-Grignon, France

J.-M. Meynard

UAR Département SAD, INRA,

Thiverval-Grignon, France

e-mail: meynard@grignon.inra.fr
}

amendment, can be used in work-like situations and then discussed during debriefing sessions among designers and users. This stage mediates dialogue between all the participants and allows them to develop cross-learning processes. We discuss how these two stages allow for a coordinated expansion of three spaces: the concept space, the knowledge space and the use space. We then discuss the need for such participatory design processes described as dialogical design processes and their contribution to produce new agronomic knowledge supporting a more sustainable agriculture. Finally, we point out a need to provide more opportunity for scientific discussion on participatory design approaches and on design methodology more broadly within the agronomic community.

Keywords Participatory design · Decision support tool · Dialogical design · Yield gap analysis · Genotype $\times$ environment interaction $\cdot$ Fungicide treatment

\section{Introduction}

As Meynard and Sebillotte (1989) pointed out, the design and development of decision support tools in agriculture has become a major objective for agronomists. More and more tools are now being offered, based primarily on advances in modelling and automated data capture in the field. These tools increasingly make use of the access to facilities afforded by the internet. Yet, although this engineering activity (design and development of decision support tools) is a crucial part of many agronomist-researchers' work, the improvement of design methodologies is rarely discussed within the agronomic community. In fact, even though numerous papers have been published about decision support systems ( 3,806 papers over a 
40-year period, according to the results of a search procedure run in the CABI database with the TOPIC request 'decision support system*'), few focus on describing and discussing design methodologies. The papers which discuss these aspects are often those which question the lack of use of the so-called 'decision support tools' by farmers, advisors or policymakers (see Boote et al. 1996; Cox 1996; Hammer et al. 2002; Matthews et al. 2002; McCown and Parton 2006, and Woodward et al. 2008). To explain the low level of use of decision support tools, these authors have often mentioned the contrast between the model offered and the way farmers make decisions, which has mostly led them to advocate participatory design methods (McCown 2002; Carberry et al. 2002; Breuer et al. 2008; Jakku and Thorburn 2010). Participation has become a very fashionable term. As Pretty pointed out several years ago (Pretty 1995), 'the term "people's participation" [is] now part of the normal language of many development agencies, including nongovernment organizations, government departments and banks [...]. It is such a fashion that almost everyone says that participation is part of their work. This has created many paradoxes. [...]. More often than not, people are asked or dragged into partaking in operations of no interest to them, in the very name of participation'. The vision of participation that we advocate here would match the one described by McCown (2001) suggesting the need for a shift from 'a design paradigm to one featuring dialogue in order to construct relevance to practice'. But in this paper, we additionally insist on the fact that dialogue is required not only to put a model into use in a decision support system, but also to design the model itself. We therefore propose that such participatory design be called a dialogical design process, and suggest some methodological insights on such a process.

Our dialogical design methodology was developed from two participatory projects on agronomic decision support tools. This methodology emphasizes two main stages: the diagnosis of uses and the setting up of an arrangement between designers and users based on the use of a prototype. We first present the two design projects from which we developed our methodology, and the two stages of this methodology. We then describe the outcomes of both stages. Before concluding, we open the discussion on some more generic considerations about design methodology and propose that the design of decision support in agriculture be based on the challenging of three main dimensions which are intertwined in the design process: (1) a new concept to develop (i.e. the first idea for a tool to be designed), (2) knowledge, notably scientific, to be incorporated into the tool, which already exists or has yet to be produced, and (3) work situations in which the tool may or may not find a use. We then discuss the idea that dialogical design is aimed at creating learning environments for both practitioners and scientists.

\section{Materials and methods}

\subsection{Agronomic tools designed in the two projects}

Our two examples concern the design of decision support tools intended to promote more environmentally friendly agriculture. The first, which we worked on from 2000 to 2004, aimed to avoid applying unnecessary fungicide treatments for sclerotinia on oilseed rape. To develop more environmentally friendly practices as regards crop disease control, CETIOM (French technical institute for the development of oil crops) decided in the 1990s to design a decision support system to avoid routine treatments for sclerotinia (sclerotinia sclerotiorum (lib.) de Bary) in oilseed rape. Whereas the disease significantly affects yields only 2 years out of 10 on average in France (Penaud and Kazmar 2001), most farmers spray routinely in regions where rape is frequent in the rotation. The objective of the new decision support system was to allow advisors or farmers to evaluate infection in the field at an early stage, before the effects of the disease were noticeable, and thus to identify situations where the treatment could be avoided. When our study began in 2000 , different tools were being designed simultaneously to respond to this need: an evaluation table of risks in the field, based on information on the cropping systems (Taverne et al. 2001); a 'petal kit' measuring the percentage of infected flowers by incubating a sample of flowers in a Petri dish (Cacérès et al. 2001; Taverne et al. 2003); and agroclimatic simulation models of the infection of rape by sclerotinia (Taverne 2001). Only the kit was tested beforehand in experimental conditions.

The second project, which we studied from 2003 to 2008, was designed to characterize winter wheat cultivars in order to identify those best suited to different agronomic situations. After being aimed mainly at increasing yields, plant breeding for winter wheat in France now includes other factors such as grain quality and disease tolerance (Lonnet 1997). Performance regarding these factors is required for a cultivar to be officially registered and commercialized. This registration is based on experimental data from a national network of trials coordinated by the GEVES (study group for cultivars and seeds). Other experimental networks exist, set up by the breeders or the R\&D bodies, to give advice on the cultivars to choose and how to grow them. To make the most of such networks of cultivar trials and to better define cultivar tolerance to certain limiting factors (nitrogen deficiency, water and heat stress, diseases, etc.), agronomists proposed a model based on yield gap analysis. This model reveals the limiting factors found in a given network. It then assesses the tolerance to these limiting factors of the different cultivars in the network (Lecomte et al. 2010), by analysing the differences in behaviour of the cultivars from one trial site to another, due to genotype-environment interactions (GEI) (Brancourt-Hulmel et al. 1999). At the beginning of our work on that project, a 
statistical model using input variables to be collected on the networks had been built and validated on several datasets. The designers planned to implement this model so that it could become a tool used by plant breeders, specialists in charge of cultivar registration and advisors, to characterize the cultivars better and to integrate GEI more fully into the assessment of cultivar performance.

In both projects, the researchers were aware that the representations of the biophysical situations they had elicited in the model they had built may differ from the representations that the practitioners would have to handle in these situations. They were likewise aware that tools introducing new information and new ways to assess this information would have to fit with current decision processes and with the purposeful and collective activity for which they were to be used. They therefore decided to develop rough prototypes whose interface or even underlying model could be reworked.

\subsection{Methodology to take account of uses when designing agronomic support tools}

Our methodology emphasizes two main stages: the diagnosis of uses and the setting up of an arrangement between designers and users based on the use of a prototype.

\subsubsection{First stage: the diagnosis of uses to analyse the users' work situations and the uses of existing tools}

Many prototyping approaches start the design process with a diagnosis in order to formulate the requirements better, mainly for the design of cropping or livestock systems (e.g. Dogliotti et al. 2003; Stoorvogel et al. 2004). This is usually an agronomic diagnosis which identifies the diversity of cropping or husbandry practices and relates them to the performance obtained (e.g., yield, qualitative variables, environmental impacts). The aim of this diagnosis is to detect the causes of failure to reach the expected performance (Doré et al. 2008); it may also suggest solutions. We suggest that such a diagnosis be completed by a 'diagnosis of uses' on the way the decision tools are applied in various work situations. This has two objectives. The first is to identify the diversity of situations in which the tools will help to solve a problem (e.g. to control a rape disease or to identify useful characteristics for new cultivars). The situation, as we understand it, includes: (1) the way potential users take their decisions when faced with a problem; (2) the constraints (technical, organizational, etc.) they may face when implementing solutions to this problem; (3) their own representation and interpretation of the biophysical processes and of their action on it. The second objective is to characterize better the uses that potential users make of the existing tools for taking such decisions. We try to identify how the users have fitted the existing tools into their decision and action processes, how the new tool will complement or replace these tools, and what should be modified to render this process more effective from the users' points of view. Note that when the Sclerotinia design project started, neither farmers nor advisors had tools to make decisions on treatments. We have therefore set out to understand the ways in which farmers and advisors operate on relatively similar problems, by studying how they use management tools to decide on treatments for wheat diseases.

This diagnosis of uses was built from semi-structured interviews with potential users of the tools under design (see Fig. 1 for details, as well as Lecomte et al. 2010 and Taverne and Cerf 2009). To characterize the diversity of uses of existing tools in our two design projects, we proposed three complementary points of view. The first is cognitive. It characterizes the diversity of ways of representing the agronomic problem. The second one concerns the diversity of ways in which the tools are used to take decisions. It is a functional point of view which combines the 'why' we use the tool with the 'how' we produce reliable and useful information with it. The third point of view allows us to take account of the constraints which the actors may experience in using the tools efficiently. Might these constraints influence the way the tool is used in a compatible way with its validity domain, or not? We call this an operational point of view. These three points of view enrich the way agronomists look at users, as they rarely take on board the cognitive dimension.

\subsubsection{Second stage: setting up an arrangement around a prototype to put into motion the concept, the knowledge and the users' work situations}

The second stage of our methodology aims at building interactions with users around prototype tools, based on the characteristics of their work situations and the assumptions about how they could use the tools, identified during the diagnosis. The challenge is to set up an arrangement which may set in motion the knowledge contained in the tool, the problems likely to be resolved by using it, and the ways of making decisions or a diagnosis. The arrangement should bring to light what may appear to be impossible or incomprehensible during the use of the tool in work situations. Successive loops might be required to settle agreements about: (1) the way to describe and to interpret the 'real world' at least in the working situations of the potential users, and (2) the concept and the way of making it operational through a coupling between the tool and its users' work situations. The setting up of the designers-users arrangement was based on two inseparable stages in our two design projects: the handling of the tool by the users in work-like situations, and the 'debriefing'.

- The handling is based on the idea of putting the users into a realistic work situation in which the prototype may be used. At this stage, the designers have to solve 


TOOL-INITIAL STATE
- Statistical model tested on
- Identifies the limiting
factors of the yield +
the cultivars to these factors
- Input variables collected

TOOL-PROTOTYPE

A software program (DIAGVAR). Input = weather + growth
data, yields, field management, accidents. Excel files.
- calculates intensity of limiting factors (LF)
- " which LFs explain yield losses + yield loss /LF. Graphs

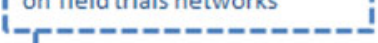$$
\text { L }
$$

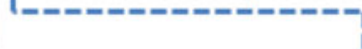

WORK CONTEXT: A strong pressure to optimize the trial networks and make more use of agronomic knowledge. A demand of public authorities to characterize cultivars according to new criteria to develop low-input cultural practices.

Semi-structured interviews : decisions to take about cultivars, tools, recent changes, difficulties 21 people from plant breeding, cultivar registration, multiplication, distribution, famers' organisation, technical institute, wheat agro-industry$$
\text { i }
$$
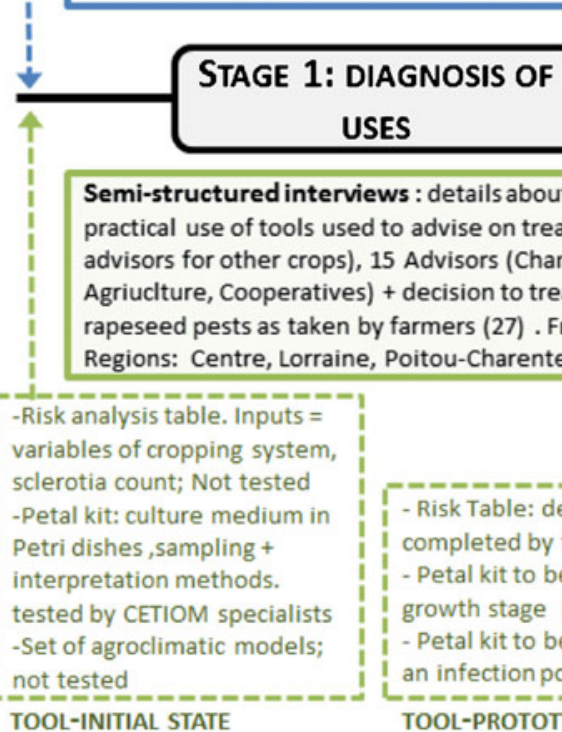

USES

the crops), 15 Advisors (Chambres A farmers (27). French egions: Centre, Lorraine, Poitou-Charentes

-Risk analysis table. Inputs $=$

For each of the 3 groups of actors ( 4 to 15 plant

METHOD

breeders, 2 to 4 advisors from technical institute, 2 specialists of registration) :

- Group training + individual use of the software for 2 -

3 weeks on the users' own databases.

- Group debriefing on what has been done + the problems encountered: work on the output produced, launching of new simulations.

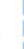

$$
\text { METHOD }
$$

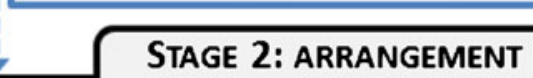

\section{AROUND A PROTOTYPE}

- In the real situation (flowering of rape, 2 regions): distribution of kits to advisors, individual debriefing by questionnaire (40 advisors each year $/ 3$ years) - In the simulated situation: presentation of the kit, use on photos (farmers). Individual debriefing ( 20 farmers: year 2001). In focus groups: presentation of use scenarios of the tools (advisors alone (10), farmers + their advisor (10)). Group discussion on the scenarios.

METHOD

THE USERS

WORK ON THEIR

PRACTICES

- Risk Table: define the infectious capacity of a field. To be
- Petal kit to be used by a farmer, on a field scale. At early
growth stage (G2).
- Petal kit to be used on a network of field trials to define
an infection potential within a field. Moment of treatment
TOOL-PROTOTYPE

WORK CONTEXT: No severe attack of sclerotinia. Resistance to carbendazime more evident. Controversy about the type of kit to be developed (based on a reaction to a fungicide or PCR kit).

Fig. 1 Details about our two design projects: description of the work context, of the tools and of the methods used for each of the two stages. At the top, cultivar assessment design project; at the bottom, sclerotinia treatment design project

the tension between developing a prototype that is complete enough to be manipulated easily, and keeping options open enough. In our two design projects, usable prototypes then had to be built (Fig. 1). The validity of their outputs was however not sufficient for them to be used in realwork situations, although it would have improved the process to be able to observe a few such situations. The observation cannot be representative, but should enable designers to grasp more fully how users apply the tools and what they say about them during the debriefings. Collective and individual sessions for handling the prototype, according to different scenarios of work situations, were then organized in our two design projects.

In the case of sclerotinia, users did not all handle the prototypes in the same way. Advisors from two regions with different rotations and treatment practices used the prototype kit and table (for details, see Fig. 1) when rape was flowering (stage G2) and before it was necessary to decide on fungicide treatments. Kits were not provided to farmers, but at meetings we showed them the kit and photos of its use, and explained how it was used, from the gathering of petals to the analysis of the results. The table was filled in during this meeting as well. In the focus groups, discussions were organized around various scenarios which each combined the stages of collection and treatment of data differently. The scenarios included various contexts of use, emphasizing most particularly the dynamics of infection and the effect of using products of varying costs to treat the crop. These methods of handling aimed at: (1) including the diversity of users and agronomic conditions during this stage; (2) bringing to light the organizational conditions to guarantee the interpretation of the results from the kit and provide timely advice to rule out the idea of treating; (3) allowing the users to assess the validity of the kit in conditions which differ from those for which its reliability had already been evaluated, and (4) weighting criteria (price of treatments, effects on the environment and on yield) in order to reveal the dependence of the intervention threshold on various combinations of these criteria. 
In the case of cultivar assessment, the Diagvar prototype computer program was tried out by three groups of people (a group of plant breeders from four different firms, one of the advisors from the French $R \& D$ institute for cereals, and one of the specialists of cultivar registration; see Fig. 1), during a quiet period for those involved, and not at the actual time when cultivars have to be evaluated. To stick as much as possible to their work situations, we proposed that the users experience the tool on a range of their own recent datasets, over a 1- to 3-week period. We asked those involved to choose, within this dataset, the combination of trials for which they wanted to do a diagnosis. We then could discuss whether the tool yielded results consistent with the limiting factors identified by the users in the trials on the basis of their own expertise. We also discussed the diversity of configurations which interested them and how this might influence the stability of the results provided by the tool.

The work of handling the tools in the two projects was usually done on an individual basis (one prototype per person), although a prior group stage was organized to present the prototypes and explain that the aim was to go back over its characteristics, be they cognitive, functional or operational. The work on the scenarios combining different actors in the sclerotinia case was organized in groups (a group of farmers only, farmers together with advisors, and advisors alone; see Fig. 1) as it aimed to discuss the organizational assumptions and to find out what was possible or unacceptable

- The debriefing allows the participants to go back over what they have done with the tool, what they did not succeed in doing, and what they would have liked to have done. It consists in looking back, mainly collectively, on the way the prototype has been used, the information it has given and its relevance, the difficulties of its use, and its advantages. It may deal with results the users did not understand, discrepancies between diagnoses made with the tool and those they would have made without it, etc. Due to the diversity of users and the places and times in which they operated, we were unable to observe directly what they did with the prototypes. They were therefore asked to note down their achievements in this respect, or the causes of difficulties or errors. However, the users generally did not have time to note down such information. We therefore organized debriefing sessions, making the best of whatever records the users did have concerning the prototype and whatever they were able to remember.

For sclerotinia, the petal kit was used by a large number of participants (50, Fig. 1). The debriefing on use of the kit and the table was done on an individual basis. We tried to find out what had been done with them, and to explore the problems that the users had been able to identify when trying them out. This exploration was done during an interview organized to discuss the tools and their use from the cognitive, functional and operational points of view. Finally, the interview ended with a discussion on changes that could be made to resolve the problems identified. In the work with focus groups the prototype was not really used, and the discussion revolved around scenarios, often with a combination of simulation and evaluation of the scenarios.

For the cultivar assessment tool, the debriefing was organized by forming groups of users working together but possibly having different occupations (for example a plant breeder and a head of cultivar development in a plant breeding firm). During the debriefings, they went over the problems they had encountered with the various datasets they chose to analyse with the prototype tool Diagvar.

\section{Results and discussion}

3.1 First stage: a way to devise new concepts for tools and to work on the flexibility required in the tools

\subsubsection{Characteristics of the use situations}

As explained in the preceding section, we used three complementary points of view to characterize the diversity of situations in which the tools may help to solve a problem, and the uses of existing tools in these situations. Table 1 shows these three points of view within each project. The study of the cognitive point of view shows significant differences between designers' and users' representations of the problem. For instance, in the case of sclerotinia, whereas the agronomists are interested in the frequency of occurrence of a severe attack (justifying a treatment), the farmers ('users') are mostly interested in avoiding situations resulting in such an attack. From a functional point of view, there is a diversity of aims and ways of producing information: for instance, in the case of cultivar assessment, the users distinguished different aims (registering, breeding, developing, range designing, indexing and scoring technological suitability), corresponding to different types of trial networks (see Lecomte et al. 2010). Finally, the operational point of view highlights the deep constraints to action experienced by each actor. In the case of sclerotinia, the farmers have to treat before the appearance of the disease, which means that farmers and advisors have little time for gathering information. In the case of cultivar assessment, the actors have little time to collect useful data and to process the data from the trials.

\subsubsection{The diagnosis of uses: a way to develop new concepts} for tools

The diagnosis of uses can help in devising new concepts for tools, by revealing ways of using existing tools, not envisaged 
Table 1 Cognitive, functional and operational points of view in the two design projects

\begin{tabular}{ll}
\hline Case study & Sclerotinia treatment \\
\hline $\begin{array}{l}\text { Cognitive point of view: diversity } \\
\text { of representations of the problem } \\
\text { between designers and users }\end{array}$ & $\begin{array}{l}\text { Users: The risk of a severe attack of sclerotinia is } \\
\text { assessed in relation to the absence of a curative } \\
\text { treatment, the severity of the damage, and the low } \\
\text { cost of the current fungicide (carbendazime) } \rightarrow \text { the } \\
\text { question for the users is not the frequency of } \\
\text { occurrence of a severe attack but rather avoiding } \\
\text { situations resulting in such an attack. }\end{array}$
\end{tabular}

Agronomists: The tools targeted the treatment of sclerotinia and do not include the possibility of complex pathogens. The risk is assessed by the frequency of occurrence of an attack to justify treatment.

Functional point of view: diversity of aims and ways of producing information

\section{In the case of wheat, the users combine different tools to identify the years, the regions and the fields at risk.}

To provide advice on the scale of a small region, advisors use the tools designed to support treatment decisions on the field scale. Some farmers use the management tools only when faced with unusual situations (see Cerf and Meynard 2006)

Farmers: In the absence of a curative treatment, they have to treat before the appearance of the disease. Most farmers do not want to spend too much time gathering information.

Advisors: They work on a network of fields (tens of $\mathrm{km}^{2}$ ) and have little time for gathering information.
Cultivar assessment (Diagvar)

Users: Most commonly, they aim to find a versatile genotype, on a scale ranging from regional to national level or even beyond. Some users aim to find a genotype which renews the available range of cultivars (quality and earliness).

Users treat the data by comparing the trials in pairs: no analysis of the overall data on the network scale.

Agronomists: They work on a network scale to extract information on the limiting factors. To assess the adaptation of the genotype to the environment, they try to analyse the GEI within the overall network.

Six different work assessment objectives were identified: registering, breeding, developing, range designing, indexing and scoring technological suitability

Nine types of cultivar trial networks were identified: registration, start/end of breeding process, indexing of company's new breeds, commercial development, seed producers, market development indexing, technical indexing and milling. (see Lecomte et al. 2010)

All the actors face strong organizational constraints: (1) to collect useful data for the interpretation of limiting factors in a network, (2) to process the data from the trials. This must be done in the period from harvest to next sowing. in their design, or by identifying problems peculiar to the users which will modify the original concept of the tool under design. Thus, in the case of sclerotinia, farmers and advisors often combine the available tools to evaluate the risk of infection over the course of time, by means of a rather sophisticated arrangement described by Cerf and Meynard (2006). These authors have developed the concept of Information and Advice Systems (IAS) to account for this arrangement between tools, advisors and farmers. Likewise, the difference in the perception of the risk, as well as the designers' wish to favour low-input agriculture, led some designers involved in the sclerotinia project to consider the possibility of coupling an insurance device to the evaluation of the level of infection by the petal kit, and thus to develop a new concept for a tool to limit the fungicide treatments.

In the second case study, discussions about the functionality of the tool brought to light a major challenge for those involved in the assessment of new cultivars: the cost of the networks and the advantage of reducing the number of trials. This appeared to be possible once more information could be extracted from each of the trials. Thus the tool was to facilitate the optimization of the networks. The diagnosis of limiting factors, by means of the tool, was used to identify the trial sites which were redundant because they had the same limiting factors and thus resulted in the same cultivar rankings.

\subsubsection{Integrating the diversity of uses: what flexibility to introduce into the tool?}

Acknowledging diversity among the cognitive, functional and operational points of view is important to foster discussions among the participants on the agricultural paradigm and on the use and user models on which the tool is based. This raises questions on the flexibility required in the tool. 'Flexible' here means that the tool produces information which will be relevant and reliable for a range of decision contexts and operating methods.

Hence, in the case of sclerotinia, in order to discuss the effect of different agricultural paradigms on the threshold for deciding to spray, we borrowed and adapted some methods from the world of medicine (see Makowski et al. 2005). 
The idea was to allow users to evaluate more efficiently the quality of different indicators (the one provided by the petal kit, the one which combines the decision grid with the petal kit, etc.) regarding the effects of the disease on the yield, according to the decision rule for fungicide application. The choice of the decision rule also determined how to treat the risks of type 1 and type 2 errors in the interpretation of an indicator of the occurrence of the disease in the field (the incidence of infected flowers). The designers first and foremost wanted the tool to allow for a reduction in the frequency of treatments (accordingly, they tried to give priority to reducing type 2 errors 'to treat even if in the end it turns out to be unnecessary'), whereas the farmers were particularly anxious not to risk having the disease (thus giving priority to reducing type 1 errors, i.e. 'not treating even if it would have been necessary to do so'). Being flexible here means getting round this difference while nevertheless keeping the key idea of avoiding unnecessary treatments.

In the case of the assessment of winter wheat cultivars, flexibility first had to address the operational diversity among future users. For example, the future users did not all collect the same data on the trials: some collected weather data and data on developmental stages, while others did not. Some data might moreover be incomplete, or the precision might not always be the same because of the organizational constraints of those doing the assessment. For example, for a growth stage record, some tried to locate the precise day of appearance of the stage whereas others went past on a given day and noted the stage reached for each cultivar. The designers thus envisaged the possibility of making up for the absence of measured data on the trial networks by introducing qualitative data obtained while the assessors were visiting the trials. Flexibility also had to address functional and cognitive diversity among users and designers. The range of objectives identified (Lecomte et al. 2010) questioned the outputs which might be presented to the users, and their precision (functional diversity). In fact, the precision required was not the same if the aim was to exclude the worst genotypes or to rank all the tested genotypes correctly. Relevant and significant information differed among the users, and the tool should have allowed this diversity to be taken into account. It also had to take on board the fact that most users tried to identify cultivars which were stable over a range of environments (which means giving priority to economic considerations), even though the tool was originally designed to identify cultivars that were suited to certain environments with well-known characteristics. This discrepancy on the way to acknowledge GIE should therefore be discussed.

\subsection{Second stage: a prototype mediating dialogue} between designers and users

Dialogue around the prototype took place in different ways. First, as Schön (1983) also found, while using the prototype the potential users dialogued with their working situations. Such dialogue enabled them to formulate claims which they would then address to the designers. Such claims were based on their experiencing the tool as well as its ability to overcome some of the problems they wanted to solve. This experience was then used by the designers to question the model, the tool and the user's decision making and practices during the debriefing sessions. Dialogue was thus established not only amongst the designers and the users (Hatchuel 1996) but also more generally between humans, artefacts and situations. Debriefing sessions create learning environments in which dialogue is supported by the way the prototype responds to the use made of it in work-like situations: what the tool can or cannot do for the users, the capabilities which the users ascribe to it, the difficulties encountered in understanding what the output means, and finally the difficulties in obtaining the input data for the tool. During the debriefings, users related the problems they had encountered and the solutions they had devised to solve them, when possible. They also spoke about their discoveries, and the way they started to change their views on how to go about decision making (the information on which they grounded their decisions, the usefulness of new information). It is therefore important for the designers to accept the fact that the knowledge introduced into the tool, the capabilities which they envisage, and the ways of using the tool will be reviewed, and that a new version of the prototype should be developed.

Debriefings are opportunities to capture discrepancies in the way participants build a representation of the problem (collect and interpret information) and their appraisal of the uncertainty they face in solving this problem. In the case of cultivar assessment, for instance, the users pointed out problems in the choice of indicators for limiting factors, in the precision needed for the input data, in the factors that the tool sorts out as limiting yield in the network, in the intensity they attributed to these factors, etc. In doing so, they discussed the assumptions made in the agronomic model underlying the tool. This was also an opportunity for the designers to point out the limitations of working on a dataset which is too homogeneous (variability is needed in the intensity of the limiting factors on a network scale if they are to be located).

The exploitation of the debriefings then enabled the participants to identify what should, or could, be initiated as regards both the tool and the users' practices. This was done by eliciting the cognitive, functional and operational points of view to which the discussions during the debriefing sessions refer.

For sclerotinia and from a functional point of view, the debriefing showed that the challenges of using tools in a local network, for combining the results in a range of agronomic situations, was not yet clear for the potential users, although they usually knew 'how it works' (Cerf and Meynard 2006). 
But anticipating the way such a network can operate remained a difficult task. From a cognitive point of view, the debriefing also allowed us to point out the lack of reliable knowledge on the protocols for testing the tool on the scale of a small region. Likewise, it was an opportunity for lending support to design concepts which had already emerged during the diagnosis of uses (insurance scheme, Information and Advice System (IAS)), and led to the identification of a new design concept, i.e. 'an indicator for better timing of treatment in relation to late infections'. It enabled the designers to further elaborate on the design concepts which had been experienced during the prototype use (for example redesign the kit use protocol). From the operational point of view, proposals emerged to facilitate the data collection (how to place the petals on the culture medium for example) or to interpret the results of the kit (photos to create a reference for classifying the colours observed in the Petri dishes). On the other hand, little emerged about what might change as regards the farming practices. In fact, the farmers lacked confidence in the results of the kit in relation to the risk they thought they were taking when skipping a treatment. As a result, they did not commit themselves as to whether they would be willing to change their farming practices, and discussions mainly focused on how risk was assessed differently among the participants.

For the cultivar testing computer program and from a cognitive point of view, the debriefing indicated numerous problems calling for knowledge development (see Table 2) but only two of them were taken into account in a new version of the tool (for details see Prost and Jeuffroy 2007; Prost et al. 2008). Indeed, compromises had to be found here between improving the model underlying the tool and allowing the users to benefit from it while being aware of its possible shortcomings. From a functional point of view, the users confirmed the value of the diagnostic stage as such, as an opportunity to optimize the network and not just a stepping stone to testing the tolerance of cultivars to limiting factors. From an operational point of view, changes were suggested by the potential users, such as modifying their experimental protocols to assess certain data not currently measured, or improving their expertise (through training or by hiring specialists in statistics and modelling). Moreover, they asked for a change in the user interface so that they could go back over the calculations of indicators of the intensity of limiting factors and the thresholds defined, in order to assess the limitations expressed by these indicators.

With hindsight, the collective nature of the debriefing appeared to have several advantages First, the users definitely felt more legitimate in questioning the designers and discussing the knowledge and hypotheses embedded in the tool. Second, in discovering what others have done or intend to do, the users questioned much more critically their own way of using the tool. Finally, the questions asked helped users to explore what others have done. The debriefing was also a way of discussing what should be taken into account for the prototype to evolve or what should be done to change the users' own situations.

\subsection{Changes in the tools and current state}

Table 2 summarizes the changes made to the tools after the analysis of the users' work situations and after the users had

Table 2 Changes in the tools and knowledge explored following the use of prototypes

\begin{tabular}{|c|c|c|}
\hline Case study & Sclerotinia treatment & Cultivar assessment (Diagvar) \\
\hline \multirow[t]{2}{*}{$\begin{array}{l}\text { Changes in the tools after having } \\
\text { analysed the users' work situations } \\
\text { and after the use of the prototypes }\end{array}$} & $\begin{array}{l}\text { Changes in sampling methods and in the } \\
\text { ergonomics of the kit. Identification of the lack of } \\
\text { precision of the table. }\end{array}$ & Changes in statistical methods \\
\hline & Development of a concept of an insurance system & $\begin{array}{l}\text { Changes in the protocol for data collection (linked } \\
\text { with the tool): in particular, the nitrogen status of } \\
\text { plants and weather data }\end{array}$ \\
\hline \multirow[t]{3}{*}{$\begin{array}{l}\text { Scientific questions which emerge } \\
\text { during the debriefing }\end{array}$} & $\begin{array}{l}\text { Protocol to test a kit-type tool for use on a regional } \\
\text { scale } \\
\text { New procedure from the medical world to evaluate } \\
\text { several risk evaluation techniques (ROC curve, } \\
\text { Makowski et al. 2005) }\end{array}$ & $\begin{array}{l}\text { Redefinition of indicators of certain limiting factors } \\
\text { to be compatible with the experimental practices } \\
\text { of potential users (e.g. reliable indicator of crop } \\
\text { nitrogen status operational in routine use-Prost } \\
\text { and Jeuffroy 2007) }\end{array}$ \\
\hline & $\begin{array}{l}\text { Procedure for managing the balance between types } \\
1 \text { and } 2 \text { errors on treatment threshold }\end{array}$ & $\begin{array}{l}\text { Research on models replacing unfeasible data } \\
\text { collection (e.g. model to predict the beginning of } \\
\text { stem elongation) }\end{array}$ \\
\hline & $\begin{array}{l}\text { Thoughts on the properties of indicators used as } \\
\text { decision support: to trigger the treatment, to } \\
\text { identify risk situations, to sort out the situations in } \\
\text { which more precise but more costly indicators } \\
\text { should be used. }\end{array}$ & $\begin{array}{l}\text { Research on statistical methods to (1) improve the } \\
\text { stability of statistical results (Prost et al. 2008), } \\
\text { (2) review the statistical methods estimating } \\
\text { relations between limiting factors and yield loss } \\
\text { (e.g. quantile regression) and (3) define the } \\
\text { criteria for judging the statistical model. }\end{array}$ \\
\hline
\end{tabular}


used prototypes of the tools. It also shows how this process enabled the researchers to identify the need to extend scientific knowledge. Note that some choices made to include the users' proposals in a new version of the tool or in the users' working environment were to some extent opportunistic taking account of the time scale of the project, the cost of achieving a solution, our ability to mobilize other researchers skilled in the new fields identified, etc. Nevertheless, we kept track of the users' suggestions and of the scientific questions that the debriefings raised.

To end this section, we briefly report on the way these decision support tools are now in use. In 2008, in the case of sclerotinia, an operational kit for use in a network (vigicolza) was developed by CETIOM (Penaud and Duroueix 2009). It is now used extensively throughout France, by advisors mainly. In the case of Diagvar, a new prototype integrating modifications to the input data, new statistical methods and a new interface, was implemented in 2009. In 2010, it was tested on existing databases by the potential users in interaction with the designers, and in 2011 by the potential users alone (the designers only interacted afterwards, to obtain feedback). It is expected to be applied in 'real-work' situations on the 2012 harvest. The four plant breeding firms are still involved, as well as the agency in charge of cultivar registration and INRA's breeders. Convinced by the results of the first tests of Diagvar on winter wheat, they have begun its adaptation to other species (sugar beet, potatoes, peas and maize). The software has been developed using an open-source language so that interested parties can take over the formalisms of the tool and adapt them to their own situation and experimental databases.

\section{Taking it further: lessons for design}

\subsection{Dialogical design is compulsory to incorporate new} theories of action in the redesign of agricultural practices

When practices are to be redesigned to face new challenges which cannot be addressed with the incumbent theory of action, decision support will be grounded in new principles which can result in new theories of action. We suggest that dialogical design of such decision tools will create opportunities for the users to start developing such new theories of action, and for designers to better identify the support that the tool can afford in making decisions based on new principles. In the case of sclerotinia control, deciding not to treat, due to environmental impacts, leads to a change of the guiding principles. The learning environments created around the use of the prototype offered opportunities to identify the obstacles to be overcome by users when developing the new practice, and the way the decision support tool could be included within the new work situation.
Participatory design has for a long time advocated for the users' legitimacy to decide on an achievable and relevant level of change. Here, the design process is meant to also recognize users' theories of action and the knowledge they have built to make sense of the 'real world' in order to achieve their objectives and goals. We therefore propose to call it a dialogical design, to recognize the need to create multi-level dialogue amongst various practitioners around a prototype, as well as dialogue between these practitioners, the prototype and the work situations, as shown by Schön (1983). A dialogical design methodology is intended to address the following questions: 'have we made realistic assumptions about the way potential users make their decisions nowadays when faced with the type of decision that the tool should support? To what extent is the underlying agricultural paradigm shared among designers and users? How can the tool improve users' ability to take effective decisions? How will this tool be used, and to what extent will this use be compatible with the conditions of its validity? Will the tool fit in with existing procedures, or will it replace them?' Our methodology involving two key stages is an attempt to answer these questions.

Based on our work in the two design projects, we address these questions via the diagnosis of uses which highlights various uses requiring flexibility if the tool is to be adapted to them. The second stage affords the opportunity for designers and users to discuss the agricultural paradigm, as well as the use and user models embedded in the prototype.

\subsection{Use of a prototype to foster a dialogical design process}

Constructing prototypes in collaboration with users and testing them so that they evolve already exists in agronomy, for the design of cropping and farming systems (Vereijken 1997; Langeveld et al. 2005; Mischler et al. 2009; Oenema et al. 2001). Nevertheless, the attention we give to users' work situations, regarding not only their soil and weather conditions and the technical operations applied, but also the cognitive, functional and operational points of view, is important if we are to take account of uses and users in the design process. This raises questions on some characteristics of the prototype and the way to create work-like situations. The first point concerns the development of the tool. As the prototype approaches the final version, it is difficult for the designer to backtrack, due to the costs and time invested. On the other hand, exploring the most operational aspects with a highly conceptual artefact (for example a non-implemented model of agronomic diagnosis in the cultivar trials) is difficult. In our two examples it was the exploration of the operational point of view, by directly using the kit or the Diagvar software, which initiated creativity at the functional or cognitive levels and led the participants to explore new functionalities, new problems to solve and the concepts 
which could contribute to this. The second point deals with the flexibility to give to a tool, starting with the prototype. Since the design continues into use, the tool created will necessarily be transformed by the user. The designer thus has to devise systems which are sufficiently flexible so that their use allows room for manoeuvre (Béguin 2007). In this search for flexibility, there is a paradox: the designer must produce a tangible and practical tool but also a flexible one. Taking into account users' work situations and their diversity through a diagnostic of use situations and through the use of a prototype is a way of equipping oneself to create this flexibility in the design process. This helps to reduce the search for flexibility to an acceptable level for the design as it specifies boundaries to action, as suggested by Vicente (1999). In most agronomic engineering activity, diagnosis is intended to identify the diversity which has to be considered (e.g. Meynard 1988; Tittonell et al. 2009), sometimes structured in typologies. Here, we propose that this diversity should be organized not only by the soil, weather and technical factors normally taken into account by agronomists, but also by the cognitive, functional and operational points of view associated with the use of tools. This suggests how the flexibility of the tool could be limited accordingly, to avoid uses which are not compatible with the model embedded in the tool.

\subsection{Design as an interacting process between concepts, knowledge and users' work situations}

On the basis of numerous studies carried out in R \& D departments of industrial firms, Hatchuel and Weil (2009) suggest that design is a key contribution in the process of creating knowledge. They argue that design requires a particular form of reasoning organized around the interaction between two spaces: that of the concept $(\mathrm{C})$, which is the idea of the innovation to be designed, and that of the knowledge (K) needed to design and specify the concept. According to them, the design process aims at enabling a coordinated expansion of these two spaces, e.g. enabling the gradual specification of the concept (or the exploration of new concepts) thanks to available knowledge and to new knowledge acquisition and use. In their work, the knowledge space includes both scientific knowledge and users' knowledge about their work situations. It does not pay attention to the well-known fact that the relevance and significance of a description of a situation on which one has to act can differ between scientists and practitioners, as many researchers have already pointed out (see for example Cerf and Meynard 2006; Hubert et al. 2009; McCown 2001). Moreover, it underestimates, from our point of view, the tangible aspects of the concept, the knowledge and the decision-making processes. Therefore, as part of our reflection on agronomic engineering, we propose to elaborate on their proposal by redefining the $\mathrm{C}$ and $\mathrm{K}$ spaces and by distinguishing a third space, which we call the $\mathrm{U}$ space (for use space). For us, the $\mathrm{C}$ space is that of the concept, understood as an idea but also as a prototype which is the tangible part of the concept and which can be handled. The $\mathrm{K}$ space is that of the scientific and technical knowledge which is built and aggregated through tangible research practices (for instance, modelling or conducting experiments or interviews). And finally, the U space gives account of users' knowledge. It includes their theories of action and their decision-making processes, as well as their tangible work situations. Separating the $\mathrm{U}$ space from the $\mathrm{K}$ and $\mathrm{C}$ spaces is a way to make explicit the necessity of dialogue among various practitioners (including the researchers who design decision support systems) on what is relevant and significant for acting in the situation, as McCown (2001) has already suggested. Moreover, recognizing the tangibility of each space is a way to make explicit the need to ground the dialogue in tangible working situations rather than handling it only at a discursive level. The purpose of our methodology is therefore to create learning environments in which the discrepancies between the theories of action of users and designers are made explicit through the handling of the prototype in work-like situations. We show that this way of organizing the dialogue has the result of expanding the concept of design and enabling further development of the prototype, as well as expanding the knowledge and practices of all the participants. Indeed, we propose a methodology that organizes the dialogue around a prototype so that it fosters a coordinated expansion of all three spaces. Table 2 shows for example how the $\mathrm{C}$ space was moved following the use of the prototypes. For instance, in the case of sclerotinia, the $\mathrm{C}$ space was expanded by new concepts such as the 'Information and Advice Systems (IAS)' (see Taverne and Cerf 2009), the 'insurance system' or 'an indicator for better timing of treatment in relation to late infections'. Next, thanks to the debriefings, which explored the cognitive, functional and operational limits encountered during the use of the prototype, we notably identified new knowledge needed on statistical methods to balance type 1 and type 2 errors. In the Diagvar case, the $\mathrm{C}$ space expanded as users acknowledged the advantages of using the tool to 'optimize a network of cultivar trials'. Knowledge was produced to test the reliability of a non-destructive test for assessing the nitrogen status of plants in a range of cropping situations similar to that encountered by the operators (Prost and Jeuffroy 2007), and to build new statistical methods to identify limiting factors in different dataset configurations (Prost et al. 2008). Several questions could not be addressed immediately but were noted for further discussion.

At this stage of our work, we cannot affirm that the two stages of our methodology are neither necessary nor sufficient to encourage the coordinated expansion of the spaces of concepts or of knowledge. Other researchers (Hatchuel et al. 2009, in the industrial world, or Groot Koerkamp and 
Bos 2008, in the agricultural domain) monitor this expansion by not only increasing creativity but also including a variety of actors. Even if further investigation is needed, our examples show that thinking about uses in practice also raises the possibility of a coordinated expansion of the spaces of concepts and knowledge.

4.4 A call for more debates about design methodology in the agronomic community

The proposals explained in this paper aim to show the value of scientific reflection on design methodologies. They are intended to go beyond the usual vision of engineering activity in agronomy, often regarded as a simple gathering of knowledge produced in the discipline. Our examples show that design involves both the application of existing knowledge and the production of new knowledge. They also show that the production and exploitation of knowledge (whether agronomic or from other disciplines) during this engineering activity would be worthy of methodological and conceptual developments. This will result in greater professionalism on this aspect of the researcher's work. They suggest that it could be useful to create opportunities, first for discussion and exchange within the agronomic community, on theoretical and methodological aspects worthy of study, and second for carrying out such activities.

\section{Conclusion}

Based on two design projects, we have developed and discussed some propositions for a new participatory process for the design of agronomic decision support tools. The added value of this methodology is to enable designers and users to interact in the joint development of the tool and the working situations. To develop the methodology, we have focused on the need to take into account the use situations of the tools being designed, including their special features and their diversity, and have proposed two stages to achieve this. The first stage aims at completing a diagnosis of uses, which is a new way to start the process of designing decision support tools. This diagnosis aims to understand the work situations in which the tools may help to solve a problem. Three points of view are used to observe and characterize the work situations: a cognitive one focused on the ways of representing a problem, a functional one focused on the tools used to take decisions in that context, and an operational one focused on the constraints of the activity in which the tools will be used. We have shown from our two case studies that such diagnosis is the first way to devise new concepts (or ideas) of tools and to rethink the flexibility to give to the tools. The diagnosis is also crucial to be able to start the second stage as it identifies the work situations in which the prototype could be used. This second stage enables the users to use the prototype in real situations and to debrief on this use. It is also unusual as it allows them to continue the design into use. Our methodology enabled the participants of our two design processes to point out difficulties encountered during use and to decide how these problems should be solved within a new design cycle.

We hope to have provided the agronomic research community with elements for reflection on a participatory design approach based on dialogue around a decision support tool, elaborating on the idea suggested by McCown (2001). We believe that the dialogue has to take place quite early in the design process so that it becomes possible to simultaneously expand the concept, the knowledge and the use spaces, e.g. expanding both the intangible and tangible dimensions of these spaces. We invite the community to develop further methodological discussions on design, since dialogical design is conducive to the dynamic production and use of agronomic knowledge.

\section{References}

Béguin P (2007) In search of a unit of analysis for designing instruments. Artifact 1:12-16. doi:10.1080/17493460600610830

Boote K, Jones J, Pickering N (1996) Potential uses and limitations of crop models. Agron J 88:704-716. doi:10.2134/ agronj1996.00021962008800050005x

Brancourt-Hulmel M, Lecomte C, Meynard JM (1999) A diagnosis of yield-limiting factors on probe genotypes for characterizing environments in winter wheat trials. Crop Sci 39:1798-1808. doi:10.2135/cropsci1999.3961798x

Breuer N, Cabrera VE, Ingram KT, Broad K, Hildebrand PE (2008) AgClimate: a case study in participatory decision support system development. Clim Change 87(3-4):385-403. doi:10.1007/ s10584-007-9323-7

Cacérès F, Jung L, Penaud A (2001) Détecter le sclérotinia du colza avec le kit petales. Oleoscope 61:20-21

Carberry PS, Hochman Z, McCown RL, Dalgliesh NP, Foale MA, Poulton PL, Hargreaves JNG, Hargreaves DMG, Cawthray S, Hillcoat N, Robertson MJ (2002) The FARMSCAPE approach to decision support: farmers', advisers', researchers' monitoring, simulation, communication and performance evaluation. Agric Syst 74(1):141-177. doi:10.1016/S0308-521X(02)00025-2

Cerf M, Meynard JM (2006) Les outils de pilotage des cultures: diversité de leurs usages et enseignements pour leur conception. Nat Sci Soc 14:19-29. doi:10.1051/nss:2006004

Cox P (1996) Some issues in the design of agricultural decision support systems. Agric Syst 52:355-381. doi:10.1016/0308521X(96)00063-7

Dogliotti S, Rossing WAH, van Ittersum MK (2003) ROTAT, a tool for systematically generating crop rotations. Eur J Agron 19:239250. doi:10.1016/S1161-0301(02)00047-3

Doré T, Clermont-Dauphin C, Crozat Y, David C, Jeuffroy M, Loyce C, Makowski D, Malézieux E, Meynard JM, Valantin-Morison M (2008) Methodological progress in on-farm regional agronomic diagnosis. A review. Agron Sustain Dev 28(1):151-161. doi:10.1051/agro:2007031

Groot Koerkamp PWG, Bos AP (2008) Designing complex and sustainable agricultural production systems: an integrated and 
reflexive approach for the case of table egg production in the Netherlands. NJAS-Wagen J Life Sci 55:113-138

Hammer G, Kropff M, Sinclair T, Porter JR (2002) Future contributions of crop modelling - from heuristics and supporting decision making to understanding genetic regulation and aiding crop improvement. Eur J Agron 18:15-31. doi:10.1016/S1161-0301(02)00093-X

Hatchuel A (1996) Coopération et conception collective: variété et crises des rapports de prescription. In: de Terssac G, Friedberg E (eds) Coopération et conception. Octarès, Toulouse, pp 101-121

Hatchuel A, Weil B (2009) C-K design theory: an advanced formulation. Res Eng Des 19(4):181-192. doi:10.1007/s00163-008-0043-4

Hatchuel A, Le Masson P, Weil B (2009) Design theory and collective creativity: a theoretical framework to evaluate KCP process. In: International Conference on Engineering Design. Stanford, USA

Hubert B, de Turckheim E, Messéan A (2009) Concevoir et construire la décision: Démarches en agriculture, agroalimentaire et espace rural. Editions Quae, Paris

Jakku E, Thorburn PJ (2010) A conceptual framework for guiding the participatory development of agricultural decision support systems. Agric Syst 103(9):675-682. doi:10.1016/j.agsy.2010.08.007

Langeveld JWA, Van Keulen H, De Haan JJ, Kroonen-Backbier BMA, Oenema J (2005) The nucleus and pilot farm research approach: experiences from The Netherlands. Agric Syst 84:227-252. doi:10.1016/j.agsy.2004.06.016

Lecomte C, Prost L, Cerf M, Meynard JM (2010) Basis for designing a tool to evaluate new cultivars. Agron Sustain Dev 30:667-677. doi:10.1051/agro/2009042

Lonnet P (1997) Les résistances variétales chez le blé. CR Acad Agric Fr 83:37-50

Makowski D, Taverne M, Bolomier J, Ducarne M (2005) Comparison of risk indicators for sclerotinia control in oilseed rape. Crop Prot 24:527-531. doi:10.1016/j.cropro.2004.10.003

Matthews R, Stephens W, Hess T, Middleton T, Graves A (2002) Applications of crop/soil simulation models in tropical agricultural systems. Adv Agron 76:31-124. doi:10.1016/S0065-2113(02)76003-3

McCown RL (2001) Learning to bridge the gap between science-based decision support and the practice of farming: evolution in paradigms of model-based research and intervention from design to dialogue. Aust J Agric Res 52(5):549-571. doi:10.1071/AR00119

McCown RL (2002) Changing systems for supporting farmers' decisions: problems, paradigms, and prospects. Agric Syst 74(1):179220. doi:10.1016/S0308-521X(02)00026-4

McCown R, Parton K (2006) Learning from the historical failure of farm management models to aid management practices. Part 2. Three systems approaches. Aust J Agric Res 57:157-172. doi:10.1071/AR05052

Meynard JM (1988) Itinéraires techniques du blé d'hiver. In: Jolivet M (ed) Pour une agriculture diversifiée. L'Harmattan, Paris, pp 49-57

Meynard JM, Sebillotte M (1989) La conduite des cultures: vers une ingénierie agronomique. Econ Rurale 192/193:35-41. doi:10.3406/ecoru.1989.3988

Mischler P, Lheureux S, Dumoulin F, Menu P, Sene O, Hopquin JP, Cariolle M, Reau R, Munier-Jolain N, Faloya V, Boizard H,
Meynard JM (2009) En Picardie, 8 fermes de grande culture engagées en Production Intégrée réduisent fortement les pesticides sans baisse de marge. Courr Environ 57:73-91

Oenema J, Koskamp GJ, Galama PJ (2001) Guiding commercial pilot farms to bridge the gap between experimental and commercial dairy farms, the project 'Cows \& Opportunities'. NJAS-Wagen J Life Sci 49:277-296

Penaud A, Duroueix F (2009) Stratégie fongicide du colza: la clé d'une protection réussie et rentable pour 2009. Perspect Agr 354:56-61

Penaud A, Kazmar M (2001) Quand le sclérotinia fait de la résistance au carbendazime. Oleoscope 61:12-14

Pretty JN (1995) Participatory learning for sustainable agriculture. World Development 23(8):1247-1263

Prost L, Jeuffroy MH (2007) Replacing the nitrogen nutrition index by the chlorophyll meter to assess wheat N status. Agron Sustain Dev 27:321-330. doi:10.1051/agro:2007032

Prost L, Makowski D, Jeuffroy MH (2008) Comparison of stepwise selection and Bayesian model averaging for yield gap analysis. Ecol Model 219:66-76. doi:10.1016/j.ecolmodel.2008.07.026

Schön D (1983) The reflexive practioner: how professionals think in action. Basic Books, New York

Stoorvogel JJ, Bouma J, Orlich RA (2004) Participatory research for systems analysis: prototyping for a Costa Rican banana plantation. Agron J 96:323. doi:10.2134/agronj2004.3230

Taverne M (2001) Des modèles pour décrire le développement du Sclérotinia. Oleoscope 61:18-19

Taverne M, Cerf M (2009) Anticiper l'usage dans un projet de conception d'un outil d'aide à la décision pour lutter contre le sclerotinia du Colza: quelles interactions entre utilisateurs et concepteurs et comment évaluer leurs apports ? In: Hubert B, de Turckheim E, Messean A (eds) Concevoir et construire la décision: démarches en agriculture, agro-alimentaire, et espace rural. Editions QUAE, Paris

Taverne M, Bammé B, Pérès A (2001) Evaluer le risque d'attaque du colza par le sclérotinia. Oleoscope 61:23-26

Taverne M, Dupeuble F, Penaud A (2003) Evaluation of a diagnostic test for sclerotinia on oilseed rape at flowering. In 11th International Rapeseed Congress. Copenhagen

Tittonell P, van Wijk MT, Herrero M, Rufino M, de Ridder N, Giller KE (2009) Beyond resource constraints - exploring the biophysical feasibility of options for the intensification of smallholder crop-livestock systems in Vihiga district, Kenya. Agric Syst 101:1-19. doi:10.1016/j.agsy.2009.02.003

Vereijken P (1997) A methodical way of prototyping integrated and ecological arable farming systems (I/EAFS) in interaction with pilot farms. Eur J Agron 7:235-250. doi:10.1016/S1161-0301(97) 00039-7

Vicente K (1999) Cognitive work analysis: toward safe productive and healthy computer-based works. Lawrence Erlbaum Associates, Mahwah NJ

Woodward S, Romera A, Beskow W, Lovatt S (2008) Better simulation modelling to support farming systems innovation: review and synthesis. New Zeal J Agr Res 51:235-252. doi:10.1080/ 00288230809510452 